\title{
Effects of hyperbaric oxygen therapy (HBO) in the treatment of severe soft tissue infections
}

Freitas Silva R., Rosa P., Melo F., Gomes A., Cabral T., Gaio-Lima C.

Hospital do Divino Espírito Santo, Ponta Delgada, Portugal

Background and Goal of Study: The Hyperbaric Medicine Service (HMS) is part of the Anesthesiology

Department of Divino Espírito Santo Hospital, where one of the few devices available in Portugal is located. A

multidisciplinary team collaborates in the consultations and follow the patients that are indicated for the treatment with hyperbaric oxygen therapy (HBO).

Necrotizing soft tissue infections, such as necrotizing fasciitis, fournier's gangrene and gangrenous cellulitis, can progress rapidly to sepsis and multiorgan failure, with very high mortality rates. This study aims to demonstrate the effects of $\mathrm{HBO}$ in patients with necrotizing soft tissue infection.

\section{Materials and Methods:}

A retrospective study was carried out in which 12 patients, referred to the Hyperbaric Medicine Service (HMS) between January 2012 and September 2014, were diagnosed with necrotizing fasciitis (7), fournier's gangrene (3) or gangrenous cellulitis (2).

\section{Results and Discussion:}

Of the 12 patientes referred, only 8 completed HBO (4 men:4 women), performing on average 18 sessions (in each session HBO at 2.4ATA with 90' $\mathrm{O}_{2}$ in 3 periods of 30 '). Three of the remaning patients, despite presenting $\mathrm{HBO}$ criteria, did not performed it because of need for ventilatory support (2) and refusal of treatment (1). One of the patients had to be excluded because of long term oncologic disease. All patients underwent surgical debridement and systemic wide-spectrum antibiotic therapy. In the patients that completed the HBO sessions, there was a favorable healing evolution with a marked improvement in the affected areas, reinforcing the benefits of blood supply with greater content in oxygen.

One patient did not completed the treatment because he was transferred to his origin country, resulting in a loss of follow-up.
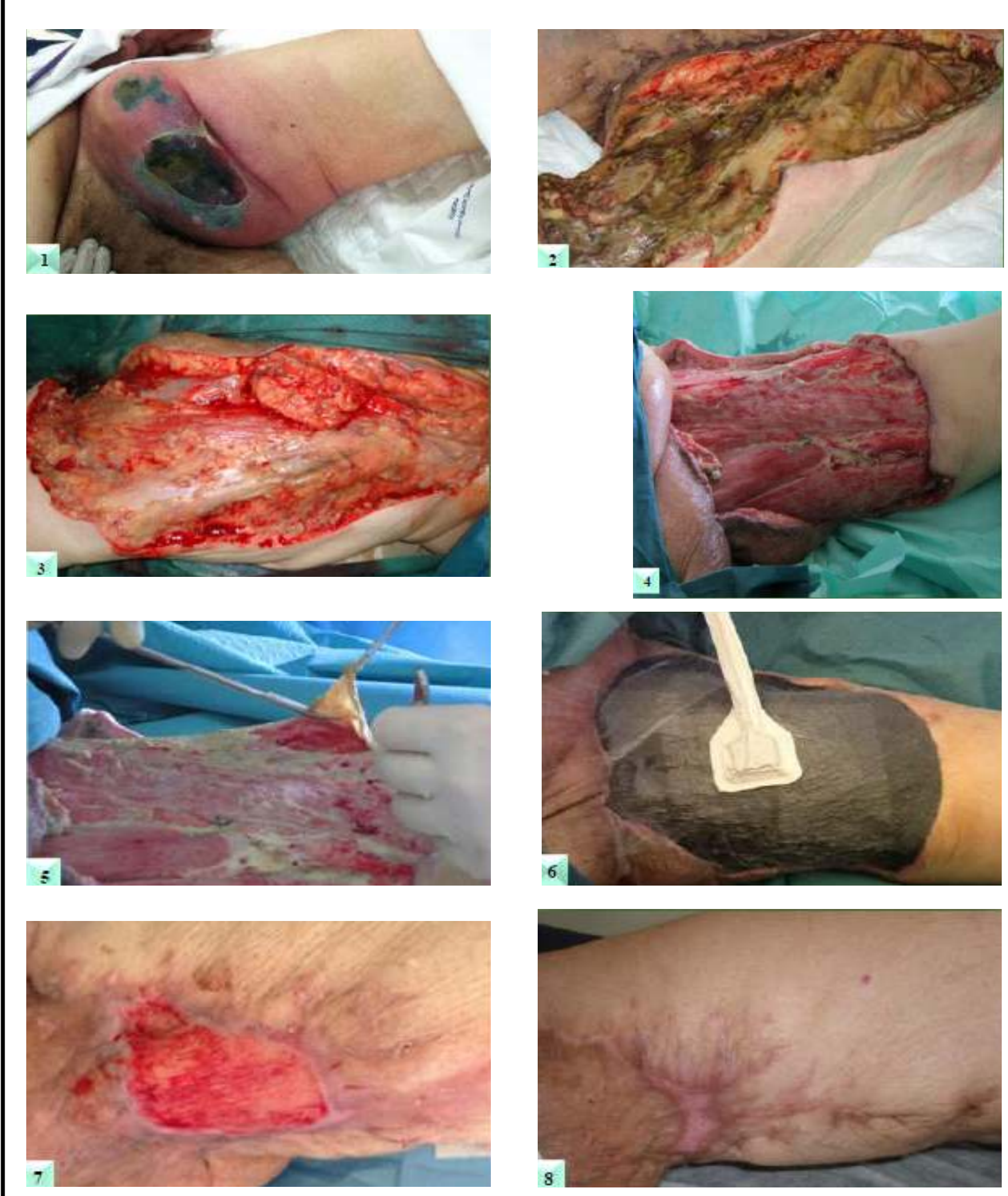

\section{Conclusion:}

A multidisciplinary approach using $\mathrm{HBO}$, accompanied by surgical debridement and adequate antibiotic therapy proved to be quite effective in controlling the pathologies in question, optimizing the conditions of local healing and systemic disease's control. The use of negative pressure and plastic surgery were advantageous for the surgical reconstruction of the affected areas. 